\title{
Stiffness-mediated adhesion of cervical cancer cells to soft hydrogel films $\dagger$
}

\author{
James P. Best, Sameen Javed, Joseph J. Richardson, Kwun Lun Cho, Marloes M. J. Kamphuis and \\ Frank Caruso*
}

\author{
${ }_{5}$ Received (in $\left.X X X, X X X\right)$ Xth $X X X X X X X X X 20 X X$, Accepted Xth XXXXXXXXX $20 X X$ \\ DOI: $10.1039 / b 000000 x$
}

We report engineered hydrogel thin-films with varying degrees of covalent crosslinking, which demonstrate enhanced HeLa cell adhesion with decreasing film stiffness. This trend is 10 contrary to previous findings for tumour cell adhesion on hydrogel substrates, and is attributed to the extremely soft nature of the films studied, allowing for a greater cell/film contact area and the development of adhesive focal contacts. Adhesion based on mechanical tuning of the film was 15 decoupled from chemical effects through characterisation and analysis of film surface roughness, hydrophobicity and charge.

Control over cellular adhesion, differentiation, migration and recolonisation through substrate modification has recently emerged as a topic of considerable research effort. ${ }^{1-5}$ ${ }_{20}$ Understanding these interactions allows for regulation of stem-cell fate ${ }^{6}$ and cell behaviour, ${ }^{7}$ leading to improved tissue scaffolds and cell adhesion-resistant coatings ${ }^{8}$ for various biomedical applications, ${ }^{2}$ or assisting circulating tumour cell (CTC) capture for efficient "liquid biopsies". 9 Microstructured hydrogel films 25 have also been utilised for drug delivery, such as in surface mediated enzyme prodrug therapy (SMEPT) ${ }^{10}$ While tuning the surface chemistry, charge, roughness, swellability, and deformability of biocompatible hydrogel networks has received the most attention, ${ }^{11-20}$ the mechanical properties of polymer

30 substrates have also been shown to have a profound effect on cellular adhesion. ${ }^{\mathbf{1 , 5}, \mathbf{1 3}-15}$

With the exception of, for example, photoreceptor and neuron cells, ${ }^{\mathbf{1 6}-17}$ most cell types preferentially adhere to, and grow on stiffer substrates., ${ }^{\mathbf{3}, 13,15,18-21}$ In particular, cancer and tumour cells 35 have been shown to adhere and migrate preferentially to more highly crosslinked hydrogel films. ${ }^{22}$ This has been demonstrated for chondrosarcoma, ${ }^{\mathbf{1 1 2 2}}$ human prostate carcinoma and glioblastoma multiforme tumour cells on various hydrogel substrates. ${ }^{23-24}$ However, due to difficulties in the fabrication and 40 characterisation of extremely soft films, predominantly planar materials with a compressive elastic modulus far exceeding $1 \mathrm{kPa}$ have been studied. ${ }^{3,13,22}$ The bulk mechanical properties of hydrogels have largely been elucidated using rotational rheological methods. $^{25}$ Atomic force microscopy (AFM), however, has 45 allowed for quantification of the mechanical properties for low Young's modulus $\left(E_{Y}<1 \mathrm{kPa}\right)$ films to be determined through small and controlled surface deformations. ${ }^{\mathbf{2 6}}$ Due to the nature of the small strain forces exerted by cells adhering to a substrate, the $E_{Y}$ is a more applicable material parameter than, for example, the 50 dynamic shear moduli obtained through bulk rotational measurements. ${ }^{26}$

Thin layer-by-layer (LbL) films have already shown promise for cell adhesion studies, ${ }^{27}$ while thiol-functionalised poly(methacrylic acid) (PMAsH) systems are promising for ${ }_{55}$ biomedical applications. ${ }^{28}$ In this context, we sought to fabricate extremely soft LbL assembled PMAsH hydrogel networks and characterise the mechanical properties by AFM, to correlate film stiffness with the adhesion of HeLa cervical cancer cells.

In order to tune the film mechanical properties, a commercial ${ }_{60}$ PMA precursor was modified with target percentages of 5, 10, 15 or $20 \mathrm{~mol} \%$ pendant-thiol functionality $(\operatorname{PMAsH}(\mathbf{x}))$, and quantified using ${ }^{1} \mathrm{H}-\mathrm{NMR}$. Single-component, disulfide crosslinked PMAsH films were prepared from 5, 10, 15 or 20 mol\% thiol-modified PMA on negatively charged mica and Thermanox substrates using 65 a monolayer of poly(ethyleneimine) (PEI) as an electrostatic binding agent. LbL PMAsH films were assembled at $\mathrm{pH} 4$ with a complementary hydrogen-bonding partner ( $\operatorname{poly}(N-$ vinylpyrrolidone), PVPON), before oxidative crosslinking and washing into phosphate buffered saline (PBS, pH 7.4), as shown in 70 Fig. 1. Due to hydrogel film instability in air, we maintained the PMAsh films in a constant state of hydration. A detailed experimental description is provided in the Supplementary Information. We first investigated the effect of PMA thiol modification $(5,10,15$ or $20 \mathrm{~mol} \%)$ upon the surface properties of 75 the films, which include surface charge, roughness, and hydrophobicity, as shown in Fig. 2. For surface charge analysis, films were assembled on $5 \mu \mathrm{m}$ diameter silica particles and the $\zeta$ potential was measured in Milli-Q water adjusted to $\mathrm{pH}$ 7.4. As shown in Fig. 2, the $\zeta$-potential of the four PMAsH films was ca. $8050 \mathrm{mV}$. The magnitude of the $\zeta$-potential when compared to bare mica and PEI-coated mica suggests that PMAsH films are present on the particles, and that there is negligible influence of the substrate or PEI monolayer on the surface charge properties.

In addition, the surface roughness of the PMAsH films was 85 evaluated using non-contact mode AFM imaging in PBS buffer. Through surface metrology analysis, the roughness ratio $(r)$ of the films was found to increase from 1.0048 to 1.0425 , with increasing PMA thiol modification. We would expect such an increase, as increasing pendant-thiol modification of the PMA backbone 90 affords slightly more hydrophobic polymer chains, giving rise to 
small hydrophobic clusters on the surface, as evidenced by AFM imaging measurements in PBS buffer (Supplementary Information). The results also indicate that all PMASH films studied are smooth, as $r \rightarrow 1$. To study the hydrophobic nature of the films, 5 an inverse captive bubble (ICB) technique was used. An air bubble was first introduced onto an inverted coated substrate, and submerged in water to determine the water contact angle of the hydrophilic surfaces. Using the Wenzel relation for evaluating the Young contact angle $\left(\theta_{\text {Young }}\right)$ for an ideal surface (Supplementary 10 Information), the hydrophobicity of the films was investigated. The Wenzel equation accounts for variations in contact angle due to surface roughness, allowing the Young's contact angle ( $\left.\theta_{\text {Young }}\right)$ for a specific surface chemistry to be determined, as shown in Fig. 2. As predicted, there was a slight increase in film hydrophobicity 15 with increasing PMA thiol modification; however, all PMAsH films were hydrophilic. It should also be noted that mica gave a Young contact angle of $c a .7^{\circ}$ corresponding well with literature values. ${ }^{29}$ Results of the film surface characterisation demonstrated that there were negligible differences in the film charge, roughness, 20 and hydrophobicity, which may influence the cell adhesion measurements.

Next, the films were characterised using an AFM force spectroscopy technique (see Supplementary Information for details). Films were indented using a colloidal probe (probe 25 diameter $c a .30 \mu \mathrm{m}$ )-modified tipless-cantilever in PBS buffer. From this indentation, force curves were generated for the films, and adjusted to subtract the effect of cantilever bending (Equation 2, Supplementary Information). As shown in Fig. 3, the film stiffness $\left(k_{f i l m}\right)$, or the initial slope of the force-deformation $(F-\delta)$ 30 curve increases substantially with increasing crosslinking. Also observed was a general film thickness of $130 \pm 50 \mathrm{~nm}$ for all PMA modified films, and that the work of adhesion $\left(W_{a d h}\right.$. ) between the film and probe decreased as the film stiffness increased. The film thickness was evaluated as the deformation distance of the probe, 35 from initial contact to substrate-incompressibility, based on a literature technique; ${ }^{30}$ however, no relationship was observed between film thickness and crosslinking density. The Hertz model is the standard model for evaluating the $E_{Y}$ of fully-elastic nonadhesive and homogeneous films; however, as the softer PMAsH 40 films have a non-zero $W_{\text {adh }}$, the Johnson-Kendall-Roberts (JKR) model was utilised. ${ }^{31}$ The JKR model assumes a strong short-range adhesive contact between film and probe, and can be used when the size of the probe is much greater than the film indentation. A representation of this is also presented in Fig. 3. The JKR model is 45 described as:

$$
\begin{aligned}
a^{3}=\frac{3 R\left(1-v^{2}\right)}{4 E_{Y}}( & F+3 W_{a d h .} \pi R \\
& \left.+\sqrt{6 W_{a d h .} \pi R F+\left(3 W_{a d h .} \pi R\right)^{2}}\right)
\end{aligned}
$$

where $a$ is the radius of the contact area. It should also be noted that for the Hertz relation, $W_{a d h} \rightarrow 0$. Using equation 2, the $E_{Y}$ for 50 the PMAsh films could be elucidated as described in the Supplementary Information.

As shown in Fig. 3, as the 20 mol\% modified PMA film is much stiffer, it is less adhesive $\left(W_{a d h .} \sim 0 \mathrm{~J}\right)$ and the Hertzian $E_{Y}$ is roughly equivalent to the JKR value. For the other films, as there is a 55 considerable $W_{a d h}$. the JKR $E_{Y}$ values are greater than their Hertzian counterparts, and based on the readily deformable nature of the films, give a better representation of the elastic modulus. Irrespective of the analysis method, it can be seen that the $E_{Y}$ increases with increasing thiol modification, as expected for an 60 increasingly densely crosslinked network.

In order to test the influence of the film mechanical properties on HeLa cell adhesion, fluorescently labelled cells were incubated with the films and bare substrates for $6 \mathrm{~h}$ in cell media at $37{ }^{\circ} \mathrm{C}$ with $5 \% \mathrm{CO}_{2}$. A $6 \mathrm{~h}$ incubation time was chosen based on ${ }_{65}$ preliminary experiments, to determine the point where the cells had initiated adhesion processes and were weakly anchored (evidenced by the development of HeLa stretching and focal adhesions). Post incubated films were washed thoroughly with PBS buffer to remove cells that had not strongly adhered to the 70 surface, and then imaged using a fluorescence microscope. At least five representative images were taken for each sample, and adhered cells were counted as shown in Fig. 4. It was found that the adhesion increased as the film stiffness and $E_{Y}$ decreased to $0.70 \mathrm{mN} / \mathrm{m}$ and $1 \mathrm{kPa}$, respectively (Fig. $4 \mathrm{a}$ ), while negligible cell 75 morphological differences were seen for the substrates studied. HeLa cell incubation experiments were reproduced in triplicate on three separate occasions. In addition, the same trends and magnitudes were observed for HeLa cells cultured on PMAsH films assembled on Thermanox substrates (Supplementary Information).

${ }_{80}$ Cells were observed to be predominantly spherical; however, some cells spread on the substrate, indicating the formation of focal contacts. Interestingly, it was also found that bare cell culture wells and Thermanox substrates $\left(E_{Y}>1 \mathrm{MPa}\right)^{23}$ demonstrated a $6 \mathrm{~h} \mathrm{HeLa}$ adhesion similar to the 20\% PMAsH film (Supplementary ${ }_{85}$ Information). The results obtained are in contrast to current literature on tumour and cancer cell adhesion substrates, where these cells tend to preferentially adhere to stiffer substrates. ${ }^{\mathbf{1 1 , 2 2 , 2 4}}$ Additionally, it had been previously found that free thiol groups on the surface of PMAsH capsules improved association between 90 capsules and HeLa cells. ${ }^{32}$ To our knowledge, stable films with a compressive elastic modulus of $c a .1 \mathrm{kPa}$ have not been examined regarding cellular adhesion. Such soft, low spring constant, films should yield considerably to static cell weight forces, giving rise to enhanced cell/film contact areas which may promote adhesion.

95 To test the influence of film spring-constant on interaction area, the deformation affected by the weight force of the cell was calculated for the PMASH films assuming a static cell on a spring, and using $k_{f i l m}$ as the spring constant. It was assumed that the model HeLa cell was not strongly adhered at $6 \mathrm{~h}$, and as such maintained 100 a spherical morphology, consistent with observations. The weight force exerted was calculated based on the average HeLa cell weight from literature (1.91 ng for a $14-16 \mu \mathrm{m}$ cell $),{ }^{33}$ and this force was offset by a buoyancy force for the cell in media. To account for film adhesive properties in JKR theory, a 'lipping' 105 factor (defined as $10 \%$ of the indentation depth) was also utilised (Fig. 4b). As such, the indentation depth of a static cell on each film was calculated, and shown to increase dramatically from 1.3 $\mathrm{nm}$ for films with $20 \mathrm{~mol} \%$ modification, to $10.9 \mathrm{~nm}$ for $5 \mathrm{~mol} \%$ modified PMAsH films. The cell/film contact area was then 110 estimated based on these calculations (Fig. 4a), where the error magnitude is based on uncertainties in the measured $k_{f i l m}$. A similar trend was observed for the contact area, as was seen for the cell adhesion (Fig. 4a). As differences in the surface charge and hydrophobicity were found to be negligible for the PMAsH films 
studied, the differences seen in cell adhesion are postulated to be solely contributed to by the film mechanical properties, considering that the experiments were performed using the same conditions. This is an analogous effect to that observed by

5 Trappmann et al., where it was found that stem cells exerted a mechanical force on collagen fibres anchored to hydrogel surfaces. ${ }^{6}$ It is possible that due to the enhanced surface area, cellular proteins are able to form more initiatory focal contacts to the substrate over the $6 \mathrm{~h}$ incubation time.

10 In summary, we have demonstrated a mechanically-tuneable hydrogel film with enhanced cancer cell adhesion properties over $6 \mathrm{~h}$. PMAsH films made from PMA with different thiol contents were fabricated using the $\mathrm{LbL}$ technique. From surface hydrophobicity, $\zeta$-potential, and AFM imaging analysis it was 15 determined that there were negligible differences in the surface chemistry of the PMAsH hydrogel films. The mechanical properties of the films could be controllably tuned by adjusting the thiol-modification percentage of the PMA backbone building blocks, and this property was found to mediate HeLa cell adhesion 20 over $6 \mathrm{~h}$. It was postulated that this effect was due to an enhanced cell/film contact area for softer PMAsH films. Super-soft hydrogel films may provide an alternate materials technology approach for applications necessitating improved short-term and non-specific cell adhesion.

\section{${ }_{25}$ Acknowledgements}

This work was supported by the Australian Research Council under the Federation Fellowship (FF0776078, F.C.), Australian Laureate Fellowship (FL120100030, F.C.) and Discovery Project (DP0877360, F.C.) schemes. The authors would also like to thank 30 Dr. Henk Dam for assistance with NMR spectroscopy measurements.

\section{Notes and references}

* Department of Chemical and Biomolecular Engineering, The University of Melbourne, Parkville, Victoria 3010, Australia. Fax: +61 38344 4153; 35 Tel: +6138344 3461; E-mail: fcaruso@unimelb.edu.au

$\dagger$ Electronic Supplementary Information (ESI) available: Polymer synthesis, ${ }^{1} \mathrm{H}-\mathrm{NMR}$ spectra for PMA-PDA, film fabrication and AFM, $\zeta$ potential, and ICB characterisation, AFM force spectroscopy measurement and data treatment, cell adhesion and control experimental techniques, and 40 $E_{Y}$ and cell/film contact area modelling. See DOI: 10.1039/b000000x/

1 P. C. Georges and P. A. Janmey, J. Appl. Phys. 2005, 98, 1547-1553.

2 S. Van Vlierberghe, P. Dubruel and E. Schacht, Biomacromolecules 2011, 12, 1387-1408.

453 C. Picart, Curr. Med. Chem. 2008, 15, 685-97.

4 J. Liu, Y. Tan, H. Zhang, Y. Zhang, P. Xu, J. Chen, Y.-C. Poh, K. Tang, N. Wang and B. Huang, Nat. Mater. 2012, 11, 734-741.

5 D. E. Discher, P. Janmey and Y.-1. Wang, Science 2005, 310, 11391143.

506 B. Trappmann, J. E. Gautrot, J. T. Connelly, D. G. T. Strange, Y. Li, M. L. Oyen, M. A. Cohen Stuart, H. Boehm, B. Li, V. Vogel, J. P. Spatz, F. M. Watt and W. T. S. Huck, Nat. Mater. 2012, 11, 642-649.

7 R. G. Wells, Hepatology 2008, 47, 1394-1400.

8 B. Cao, S. Yan, K. Zhang, Z. Song, X. Chen, L. Cui and J. Yin,

55 Macromol. Biosci. 2011, 11, 1211-1217.

9 S. Wang, K. Liu, J. Liu, Z. T. F. Yu, X. Xu, L. Zhao, T. Lee, E. K. Lee, J. Reiss, Y.-K. Lee, L. W. K. Chung, J. Huang, M. Rettig, D. Seligson,
K. N. Duraiswamy, C. K. F. Shen and H.-R. Tseng, Angew. Chem. Int Ed. 2011, 50, 3084-3088.

6010 B. Fejerskov, B. E. B. Jensen, N. B. S. Jensen, S. F. Chong and A. N. Zelikin, ACS Appl. Mater. Interfaces 2012, 4, 4981-4990.

11 L. Richert, Y. Arntz, P. Schaaf, J.-C. Voegel and C. Picart, Surf. Sci. 2004, 570, 13-29.

12 K. Trenkenschuh, J. Erath, V. Kuznetsov, J. Gensel, F. Boulmedais, P.

65 Schaaf, G. Papastavrou, A. Fery, Macromolecules 2011, 44, 89548961.

13 A. J. Engler, L. Richert, J. Y. Wong, C. Picart and D. E. Discher, Surf. Sci. 2004, 570, 142-154.

14 T. Yeung, P. C. Georges, L. A. Flanagan, B. Marg, M. Ortiz, M.

70 Funaki, N. Zahir, W. Ming, V. Weaver and P. A. Janmey, Cell Motil. Cytoskel. 2004, 60, 24-34.

15 C. Monge, N. Saha, T. Boudou, C. Pózos-Vásquez, V. Dulong, K. Glinel and C. Picart, Adv. Funct. Mater. 2013, DOI: 10.1002/adfm.201203580.

7516 A. P. Balgude, X. Yu, A. Szymanski and R. V. Bellamkonda, Biomaterials 2001, 22, 1077-1084.

17 A. Tezcaner, D. Hicks, F. Boulmedais, J. Sahel, P. Schaaf, J. C. Voegel and P. Lavalle, Biomacromolecules 2006, 7, 86-94.

18 C.-M. Lo, H.-B. Wang, M. Dembo and Y.-1. Wang, Biophys. J. 2000, $80 \quad$ 79, $144-152$

19 C.-H. R. Kuo, J. Xian, J. D. Brenton, K. Franze and E. Sivaniah, Adv. Mater. 2012, 24, 6059-6064.

20 S. Schmidt, M. Zeiser, T. Hellweg, C. Duschl, A. Fery, H. Möhwald, Adv. Funct. Mater. 2010, 20, 3235-3243.

8521 S. Schmidt, N. Madaboosi, K. Uhlig, D. Köhler, A. Skirtach, C. Duschl, H. Möhwald, D. V. Volodkin, Langmuir 2012, 28, 7249-7257.

22 A. Schneider, G. Francius, R. Obeid, P. Schwinté, J. Hemmerlé, B. Frisch, P. Schaaf, J. C. Voegel, B. Senger and C. Picart, Langmuir 2006, 22, 1193-1200.

9023 S. S. Rao, S. Bentil, J. DeJesus, J. Larison, A. Hissong, R. Dupaix, A. Sarkar and J. O. Winter, PLoS ONE 2012, 7, e35852.

24 M. H. Zaman, L. M. Trapani, A. L. Sieminski, D. MacKellar, H. Gong, R. D. Kamm, A. Wells, D. A. Lauffenburger and P. Matsudaira, Proc. Nat. Acad. Sci. U.S.A. 2006, 103, 10889-10894.

9525 I. Levental, P. C. Georges and P. A. Janmey, Soft Matter 2007, 3, 299306.

26 A. J. Engler, F. Rehfeldt, S. Sen and D. E. Discher, Methods in Cell Biology, ed. W, Yu-Li and D. E. Discher, Academic Press, Massachusetts, 2007, vol. 83, pp. 521-545.

10027 Z. Tang, Y. Wang, P. Podsiadlo and N. A. Kotov, Adv. Mater. 2006, 18, 3203-3224.

28 S. F. Chong, J. H. Lee, A. N. Zelikin and F. Caruso, Langmuir 2011, 27, 1724-1730.

29 R. Kohli and K. L. Mittal, Contamination and Cleaning -

105 Fundamentals and Applied Aspects, William Andrew, London, 2008 ch. 13, p. 738 .

30 C. E. McNamee, S. Yamamoto and K. Higashitani, Langmuir 2007, 23, 4389-4399.

31 K. L. Johnson, K. Kendall and A. D. Roberts, Proc. R. Soc. Lond. A. 1971, 324, 301-313

32 Y. Yan, Y. Wang, J. K. Heath, E. C. Nice and F. Caruso, Adv. Mater. 2011, 23, 3916-3921. 
33 R. I. Freshney, Culture of Animal Cells: A Manual of Basic Technique and Specialized Applications, Wiley, New York, 2011. 


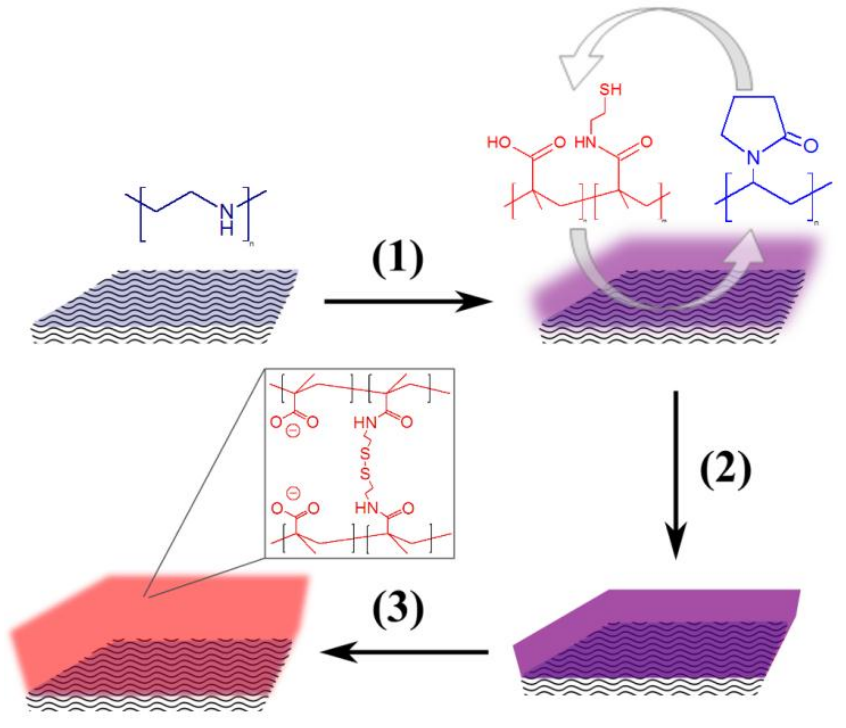

Fig. 1. Schematic of PMAsH film fabrication onto PEI-coated substrates. PMAsH (red) and PVPON (blue) are assembled via the LbL technique (five bilayers) at $\mathrm{pH} 4.0$ (1) before oxidative crosslinking using chloramine-T (2). Films are washed into PBS buffer (pH 7.4) to remove unbound PVPON (3), ${ }_{5}$ yielding single-component crosslinked $\mathrm{PMA}_{\mathrm{SH}}$ films (inset). 

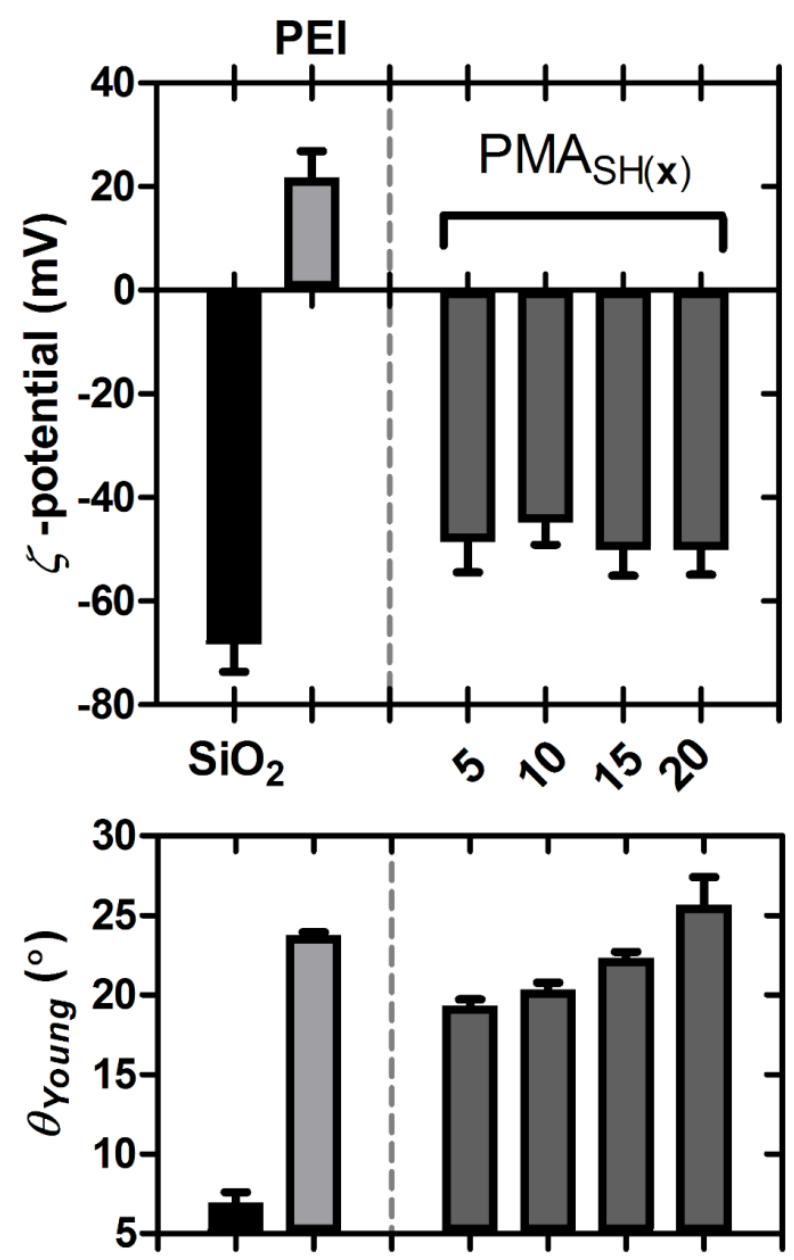

Fig. 2. Zeta-potential ( $\zeta$, top) and Young contact angle $\left(\theta_{\text {Young }}\right.$, bottom) for bare silica substrates, PEI adsorbed silica, and PMAsh films prepared from PMA with varying degrees of thiolation $(5,10,15$ and $20 \mathrm{~mol} \%$ ) on PEI-coated silica. Error bars represent one standard deviation. 

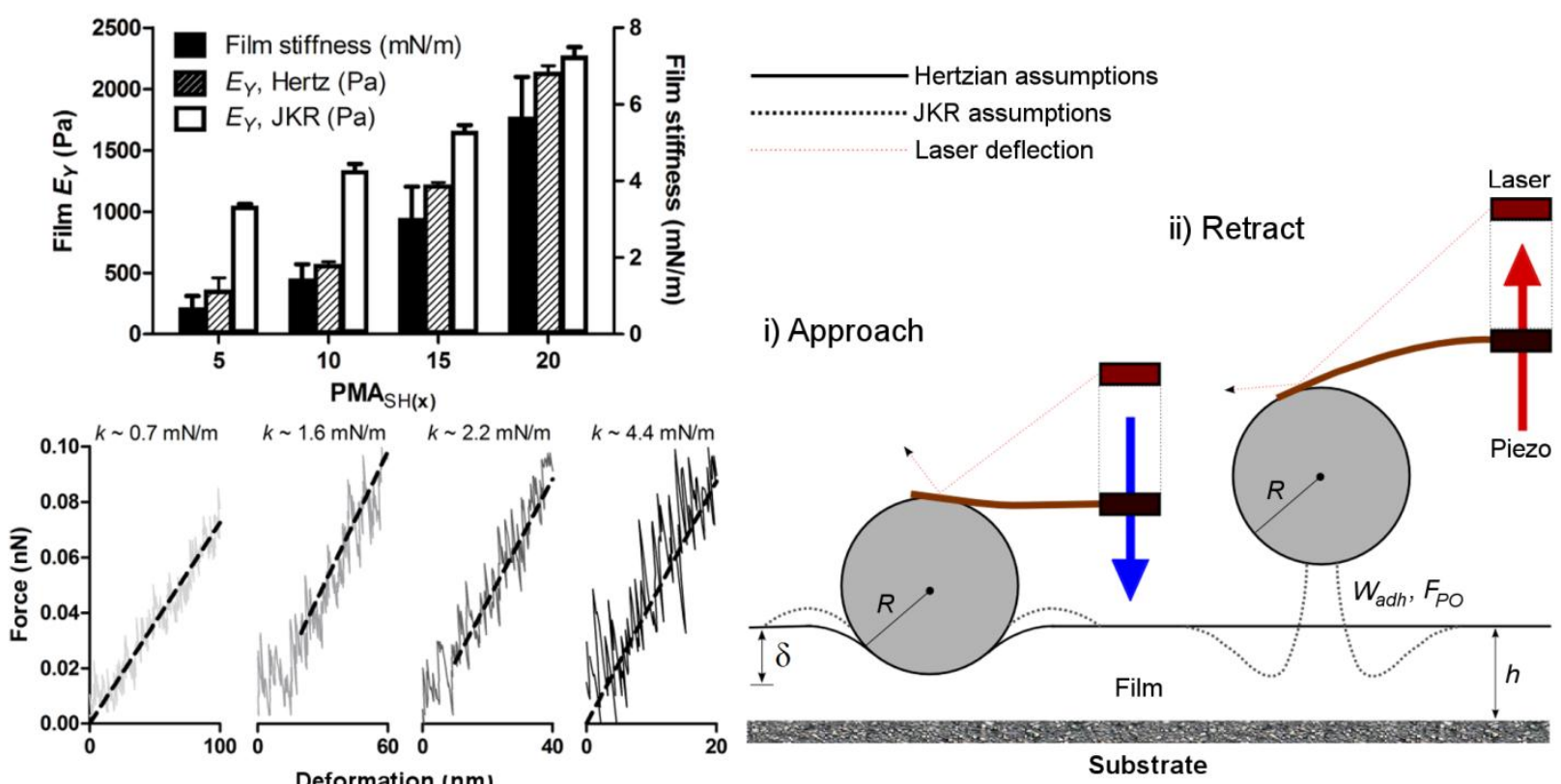

Fig. 3. Stiffness and Young's modulus (Hertz and JKR modelled) results, with representative $F-\delta$ curves with linear stiffness fits, for PMASH films with various PMA thiol content (left). Schematic representation (right) of colloidal-probe AFM indentation of soft films, where expected deformations ${ }_{s}$ according to both Hertz and JKR theory are shown. Error bars for $k_{f i l m}$ and $E_{Y}$ results represent one standard deviation. 

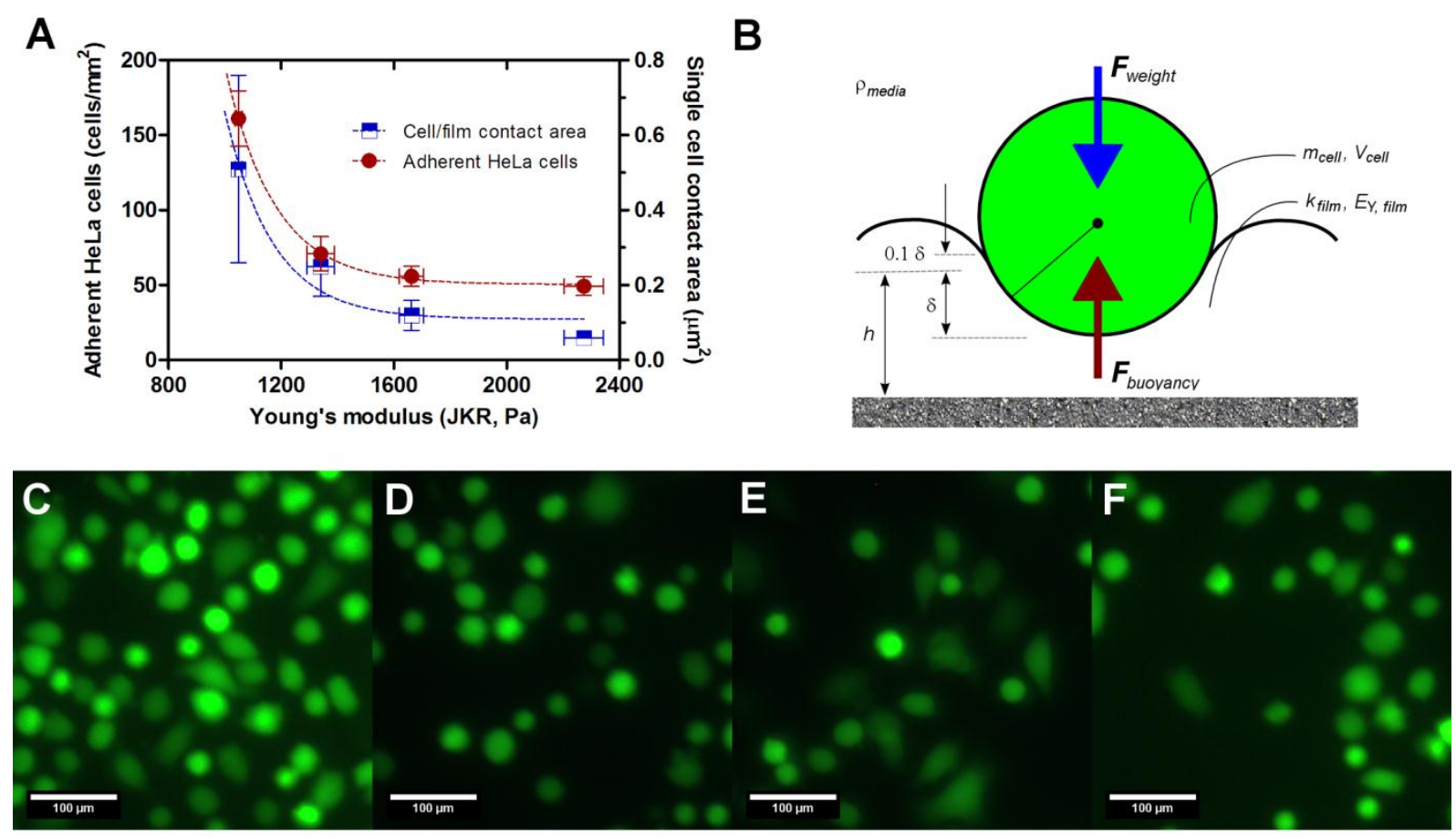

\section{Increasing film $E_{Y} /$ stiffness}

Fig. 4. HeLa cell adhesion and cell contact area as a function of Young's modulus (A), where the cell/film contact area was modelled based on the assumptions depicted in $\mathbf{B}$. The curves in $\mathbf{A}$ are to guide the eye only. Fluorescence microscopy images $\mathbf{C}, \mathbf{D}, \mathbf{E}$ and $\mathbf{F}$ show adhered HeLa cells on PMA $\mathrm{SH}(\mathbf{x})$ ${ }_{5}$ films (5, 10, 15 and 20 mol\% respectively) after washing. Error bars in A represent one standard deviation. 